\title{
Prenatal Nicotine Exposure Alters Central Cardiorespiratory Responses to Hypoxia in Rats: Implications for Sudden Infant Death Syndrome
}

\author{
Robert A. Neff, ${ }^{1}$ Samuel J. Simmens, ${ }^{2}$ Cory Evans, ${ }^{1}$ and David Mendelowitz ${ }^{1}$ \\ ${ }^{1}$ Department of Pharmacology and Physiology and ${ }^{2}$ Center for Biostatistics, Medical Center Unit, The George Washington University, \\ Washington, DC 20037
}

\begin{abstract}
Maternal cigarette smoking and prenatal nicotine exposure are the highest risk factors for sudden infant death syndrome (SIDS). During hypoxia, respiratory frequency and heart rate transiently increase and subsequently decrease. These biphasic cardiorespiratory responses normally serve to prolong survival during hypoxia by reducing the metabolic demands of cardiac and respiratory muscles. However, exaggerated responses to hypoxia may be life threatening and have been implicated in SIDS. Heart rate is primarily determined by the activity of brainstem preganglionic cardioinhibitory vagal neurons (CVNs) in the nucleus ambiguus. We developed an in vitro rat brainstem slice preparation that maintains rhythmic inspiratory-related activity and contains fluorescently labeled CVNs. Synaptic inputs to CVNs were examined using patch-clamp electrophysiological techniques. Hypoxia evoked a biphasic change in the frequency of both GABAergic and glycinergic IPSCs in CVNs, comprised of an initial increase followed by a decrease in IPSC frequency. Prenatal exposure to nicotine changed the GABAergic response to hypoxia from a biphasic response to a precipitous decrease in spontaneous GABAergic IPSC frequency. This study establishes a likely neurochemical mechanism for the heart rate response to hypoxia and a link between prenatal nicotine exposure and an exaggerated bradycardia during hypoxia that may contribute to SIDS.
\end{abstract}

Key words: nucleus ambiguus; cardiac; vagal activity; hypoxia; nicotine; sudden infant death syndrome; GABA

\section{Introduction}

Hypoxia evokes profound cardiovascular and respiratory responses. Hypoxia initially elicits an increase, followed by a decrease, in respiratory frequency. In addition, hypoxia transforms eupnic respiratory activity to gasping activity, which is characterized by infrequent, robust breaths of short duration (Guntheroth and Kawabori, 1975; Gozal et al., 2002). Prolonged hypoxia eventually produces a terminal apnea. Likewise, hypoxia evokes an initial increase in heart rate followed by a parasympathetically mediated bradycardia and ultimately, cessation of cardiac contractions (Taylor and Butler, 1982; Schuen et al., 1997; Deshpande et al., 1999).

The reduction in heart rate and respiratory frequency in response to hypoxia normally serves to reduce the metabolic demand of the cardiac and respiratory muscles and thus prolong survival (Schuen et al., 1997). Exaggeration of this protective response to hypoxia, however, could be detrimental. Sudden infant death syndrome (SIDS) is the leading cause of infant death in

Received March 10, 2004; revised Sept. 1, 2004; accepted Sept. 2, 2004.

This study was supported by National Institutes of Health-National Heart Lung and Blood Institute Grants HL-72006 and HL-59895 to D.M. The Jocelyn Beard Moran Memorial Fellowship from the American Heart Association, Mid-Atlantic affiliate, supported R.A.N. The technical assistance of Sunit Baxi, Allison Gold, Muralikrishna Mukkamala, and Alan Zarrinneshan is gratefully acknowledged.

Correspondence should be addressed to Robert Neff, Department of Pharmacology and Physiology, The George Washington University, 2300 Eye Street Northwest, Washington, DC 20037. E-mail: phmdxm@gwumc.edu.

DOI:10.1523/JNEUROSCI.1918-04.2004

Copyright $\odot 2004$ Society for Neuroscience $\quad 0270-6474 / 04 / 249261-08 \$ 15.00 / 0$ the postneonatal period (Anderson, 2002). Infants that succumb to SIDS typically experience a severe bradycardia that precedes or is accompanied by a centrally mediated life-threatening apnea (Meny et al., 1994; Nachmanoff et al., 1998; Poets et al., 1999; Fewell et al., 2001). Although the causes of the apnea and bradycardia prevalent in SIDS victims are unknown, it has been hypothesized that these fatal events are exaggerated cardiorespiratory responses to hypoxia (Meny et al., 1994; Hunt, 2001).

Maternal cigarette smoking is highly correlated with SIDS. Nicotine has been proposed to be the link between maternal smoking and SIDS (Slotkin et al., 1997; Nachmanoff et al., 1998; Bamford and Carroll, 1999; St-John and Leiter, 1999). During hypoxia, rats exposed to nicotine prenatally become apneic more rapidly than unexposed animals (Fewell et al., 2001). In addition, neonatal rats exposed to nicotine in utero show reduced tolerance for hypoxia caused by impaired heart rate control (Slotkin et al., 1997).

Heart rate is primarily determined by the activity of brainstem preganglionic cardioinhibitory vagal neurons (CVNs) in the nucleus ambiguus (NA) (Loewy and Spyer, 1990). CVNs are intrinsically silent and thus rely on synaptic inputs to dictate their activity (Mendelowitz, 1996). CVNs receive spontaneous GABAergic (Wang et al., 2001) and glycinergic IPSCs, both of which are facilitated by the activation of nicotinic receptors (Wang et al., 2003). Recent work has demonstrated that the frequency of both GABAergic and glycinergic IPSCs in CVNs increases during inspiration and that the respiratory-related 
GABAergic, but not glycinergic, inhibition of CVNs is dependent on the activation of nicotinic receptors. In addition, prenatal nicotine $(\mathrm{PN})$ significantly enhances the inspiratory-related increase in GABAergic IPSC frequency (Neff et al., 2003).

However, the cellular bases for hypoxia-induced changes in cardiorespiratory interactions and whether these mechanisms are altered by prenatal nicotine exposure are unknown. In this study, we tested the hypothesis that hypoxia alters the frequency of spontaneous and inspiratory-related IPSCs in CVNs. Because prenatal exposure to nicotine may be critical in the pathophysiology of SIDS and nicotinic receptors are essential for the inhibition of CVNs during inspiration, we also tested the hypothesis that prenatal nicotine alters hypoxia-induced changes in GABAergic and glycinergic IPSC frequency in CVNs.

\section{Materials and Methods}

Fluorescent labeling of CVNs and medullary slice preparation. Neonatal Sprague Dawley rats [postnatal day 3-7 (P3-P7); Hilltop, Scottdale, PA] were anesthetized with ketamine-xylazine $(87 / 13 \mathrm{mg} / \mathrm{kg})$ and cooled to $\sim 4^{\circ} \mathrm{C}$ to slow the heart rate. A right thoractomy was performed, and the retrograde fluorescent tracer X-rhodamine-5-(and-6)-isothiocyanate (Molecular Probes, Eugene, OR) was injected into the fat pads at the base of the heart. After 24-48 hr recovery, animals were anesthetized with halothane, killed by cervical dislocation, and the brain tissue was placed in a $4^{\circ} \mathrm{C}$ physiological saline solution containing the following (in $\mathrm{mmol} /$ 1): $140 \mathrm{NaCl}, 5 \mathrm{KCl}, 2 \mathrm{CaCl}_{2}, 5$ glucose, and 10 HEPES, bubbled with $100 \% \mathrm{O}_{2}, \mathrm{pH}$ 7.4. All animal procedures were performed with the approval of the Animal Care and Use Committee of The George Washington University in accordance with the recommendations of the panel on euthanasia of the American Veterinary Medical Association and the $\mathrm{Na}$ tional Institutes of Health Guide for the Care and Use of Laboratory Animals. The medulla was removed with care to preserve the hypoglossal cranial nerve rootlet. The medulla was mounted on a cutting block and placed into a vibrating blade microtome (Leica, Nussloch, Germany). Serial transverse sections were sliced in a rostrocaudal progression until the inferior olives and the nucleus ambiguus could be visualized on the rostral surface of the tissue. A single thick $(800 \mu \mathrm{m})$ section that included CVNs, the hypoglossal nerve rootlet, the pre-Bötzinger complex, and the rostral portion of the hypoglossal nucleus was cut and submerged in a recording chamber that allowed perfusion $(4 \mathrm{ml} / \mathrm{min})$ above and below the slice with room temperature artificial CSF (aCSF) containing (in mmol/l): $125 \mathrm{NaCl}, 3 \mathrm{KCl}, 2 \mathrm{CaCl}_{2}, 26 \mathrm{NaHCO}_{3}, 5$ glucose, and 5 HEPES, equilibrated with $95 \% \mathrm{O}_{2}-5 \% \mathrm{CO}_{2}, \mathrm{pH}=7.4$.

Recording respiratory network activity. The thick medullary slice preparation generates rhythmic inspiratory-related motor discharge in hypoglossal cranial nerves (Smith et al., 1991). Spontaneous respiratoryrelated activity was recorded by monitoring motorneuron population activity from hypoglossal nerve rootlets using a suction electrode. Hypoglossal rootlet activity was amplified (50,000 times) and filtered (10-300 $\mathrm{Hz}$ bandpass; CWE, Ardmore, PA) and electronically integrated $(\tau=50$ msec; CWE).

Measurement of the pH profile of the thick medullary slice. Tissue $\mathrm{pH}$ was recorded using a $\mathrm{pH}$ microelectrode (model 823) with an external reference electrode (model 401; Diamond General, Ann Arbor, MI) and an Accumet pH meter (model AB15; Fisher Scientific, Houston, TX). Two profiles were recorded for each slice: the first while the slice was perfused with control aCSF (equilibrated with $95 \% \mathrm{O}_{2}-5 \% \mathrm{CO}_{2}$ ) and the second after the slice had been perfused with hypoxic aCSF (equilibrated with $75 \% \mathrm{~N}_{2}-20 \% \mathrm{O}_{2}-5 \% \mathrm{CO}_{2}$ ) for $10 \mathrm{~min}$. The $\mathrm{pH}$ microelectrode was calibrated, placed in a micromanipulator, and positioned above the rostral surface of the tissue. The electrode tip was then advanced in $23 \mu \mathrm{m}$ increments every $10 \mathrm{sec}$, and $\mathrm{pH}$ was recorded at each depth.

Measurement of the $\mathrm{pO}_{2}$ profile of the thick medullary slice. Tissue $\mathrm{pO}_{2}$ was recorded using a Clark-style oxygen-sensitive microelectrode (10 $\mu \mathrm{m}$ tip) and a Chemical Microsensor polarographic amplifier (model 1201; Diamond General). The oxygen microelectrode was calibrated, placed in a micromanipulator (Narishige, Tokyo, Japan), and the elec- trode tip was manipulated to the rostral surface of the tissue. The electrode was advanced to increasing depths in $25 \mu \mathrm{m}$ increments every 10 sec. $\mathrm{pO}_{2}$ was recorded at each depth. Profiles were generated for slices perfused with both control (equilibrated with $95 \% \mathrm{O}_{2}-5 \% \mathrm{CO}_{2} ; n=7$ ) and hypoxic, isocapnic aCSF (perfused for $10 \mathrm{~min}$ with aCSF equilibrated with $75 \% \mathrm{~N}_{2}-20 \% \mathrm{O}_{2}-5 \% \mathrm{CO}_{2} ; n=6$ ).

Time course of tissue $\mathrm{pO}_{2}$ changes during hypoxia. To examine the time course of the change in $\mathrm{pO}_{2}$ levels in experiments involving hypoxia, seven additional experiments were performed in which the tip of the electrode was placed at a depth of $300 \mu \mathrm{m}$ beneath the surface of the slice. Tissue $\mathrm{pO}_{2}$ was then recorded every 15 sec during control (4 min while bathing the slice in aCSF equilibrated with $95 \% \mathrm{O}_{2}-5 \% \mathrm{CO}_{2}$ ), hypoxia (10 min in aCSF equilibrated with $75 \% \mathrm{~N}_{2}-20 \% \mathrm{O}_{2}-5 \% \mathrm{CO}_{2}$ ), and recovery periods ( $10 \mathrm{~min}$ in aCSF equilibrated with $95 \% \mathrm{O}_{2}-5 \% \mathrm{CO}_{2}$ ). The period of hypoxia was then defined as the time in which $\mathrm{pO}_{2}$ was $\leq 23$ mm Hg (Doppenberg et al., 1998). The period between the time the slice began to be perfused with hypoxic media but before $\mathrm{pO}_{2}$ dropped below the hypoxic threshold was defined as a transition phase.

Patch-clamp techniques. CVNs in the NA were identified by the presence of the fluorescent tracer, as described previously (Mendelowitz and Kunze, 1991; Mendelowitz, 1996). Briefly, slices were viewed with infrared illumination and differential interference optics (Zeiss, Oberkochen, Germany) and under fluorescent illumination with an infrared-sensitive cooled charged-coupled device camera (Photometrics, Tucson, AZ). Neurons containing the fluorescent tracer were identified by superimposing the fluorescent and infrared images on a video monitor (Sony, Tokyo, Japan).

Patch pipettes (2.5-3.5 M $\Omega$ ) were visually guided to the surface of individual CVNs using differential interference optics and infrared illumination (Zeiss). Patch pipettes contained (in mmol/l): $150 \mathrm{KCl}, 4$ $\mathrm{MgCl}_{2}, 2$ EGTA, $2 \mathrm{Na}$-ATP, and 10 HEPES, $\mathrm{pH}=7.4$. This pipette solution causes the $\mathrm{Cl}^{-}$current induced by the activation of GABA or glycine receptors to be recorded as an inward current (calculated reversal potential of $\mathrm{Cl}^{-}=+4 \mathrm{mV}$ ). Voltage-clamp recordings were made with an Axopatch 200B and pClamp 8 software (Axon Instruments, Union City, CA). All synaptic activity in CVNs was recorded at $-80 \mathrm{mV}$. Only preparations in which synaptic activity increased in CVNs during inspiration (in 71 of 82 or $87 \%$ of the preparations) were used for additional experimentation and analysis. Only one experiment was conducted per preparation.

Focal drug application. Focal drug application was performed using a pneumatic picopump pressure delivery system (WPI, Sarasota, FL). Drugs were ejected from a patch pipette positioned within $30 \mu \mathrm{m}$ from the patched CVN. The maximum range of drug application has been determined previously to $100-120 \mu \mathrm{m}$ downstream from the drug pipette and considerably less behind the drug pipette (Wang et al., 2002). GABAergic neurotransmission was isolated by focal application of D-2amino-5-phosphonovalerate (AP-5) (50 $\mu \mathrm{mol} / \mathrm{l})$, 6-cyano-7nitroquinoxaline-2, 3-dione (CNQX) (50 $\mu \mathrm{mol} / \mathrm{l})$, and strychnine (1 $\mu \mathrm{mol} / \mathrm{l})$ to block NMDA, non-NMDA, and glycinergic receptors, respectively. Glycinergic neurotransmission was isolated by focal application of AP-5, CNQX, and gabazine ( $25 \mu \mathrm{mol} / \mathrm{l})$ to block NMDA, non-NMDA, and $\mathrm{GABA}_{\mathrm{A}}$ receptors, respectively. All drugs were obtained from Sigma (St. Louis, MO).

Hypoxia. Rhythmic inspiratory-related activity and the GABAergic or glycinergic IPSCs in a single CVN were recorded simultaneously for 4 min in aCSF equilibrated with $95 \% \mathrm{O}_{2}-5 \% \mathrm{CO}_{2}$. Slices were then exposed to hypoxic, isocapnic aCSF (equilibrated with $75 \% \mathrm{~N}_{2}-20 \%$ $\mathrm{O}_{2}-5 \% \mathrm{CO}_{2}$ ) for $10 \mathrm{~min}$ and then returned to the original perfusate for $30 \mathrm{~min}$. At the end of each experiment, GABAergic or glycinergic activity was reversibly inhibited using gabazine $(25 \mu \mathrm{M})$ or strychnine $(1 \mu \mathrm{M})$, respectively.

Prenatal nicotine exposure. Adult female rats were anesthetized with ketamine-xylazine (87/13 mg/kg, i.p.; Phoenix Pharmaceuticals, St. Joseph, MO) on the third day of gestation and implanted with Alzet osmotic minipumps (Durect, Cupertino, CA) containing (-) nicotine $(56.1 \mathrm{mg} / \mathrm{ml}$ bacteriostatic saline; Sigma). Osmotic minipumps were chosen to avoid the high plasma nicotine concentrations and subsequent episodic fetal hypoxia-ischemia that can be produced by nicotine injec- 
tions (Slotkin, 1998). Pumps delivered $2.1 \mathrm{mg}$ nicotine/d, a level approximately equivalent to those that occur in moderate to heavy smokers, for 28 d (Slotkin et al., 1997).

Data analysis. Mean $\mathrm{pH}$ and $\mathrm{pO}_{2}$ values were computed for each depth in control and hypoxic slices. $\mathrm{pH}$ values were compared at both the surface and the core of the slice under control and hypoxic conditions. Statistical comparison between the $\mathrm{pH}$ of the control and hypoxic slices was made using paired Student's $t$ tests. All $\mathrm{pH}$ data are presented as means \pm SEM.

Respiratory and synaptic events in CVNs were analyzed in successive 1 min periods throughout each experiment. Respiratory frequency was defined as the total number of inspiratory bursts during each minute over the entire course of each experiment. Respiratory duration was determined throughout each experiment by dividing the sum of the duration of all inspiratory bursts in a 1 min period by the number of bursts during that minute period. To summarize the data from multiple experiments, mean frequency and duration were calculated for each time point. Because of skewed distributions, inspiratory burst frequency and duration were log-transformed, producing approximate normal distributions before the application of statistical tests.

Synaptic events were detected using MiniAnalysis version 5.6.12 (Synaptosoft, Decatur, GA). The frequency of IPSCs that occurred in CVNs during inspiratory-related hypoglossal activity was determined by dividing the number of IPSCs that occurred during the inspiratory-related discharge by the duration of the inspiratory burst. Spontaneous IPSC frequencies were determined by dividing the number of IPSCs that occurred in a 5-10 sec period ending $1 \mathrm{sec}$ before the onset of each inspiratory burst and beginning at least $3 \mathrm{sec}$ after the end of any previous inspiratory activity by the duration of this period. All IPSC data are presented as means \pm SEM. Because of skewed distributions, the frequency of glycinergic and GABAergic were log-transformed, producing approximate normal distributions before the application of statistical tests.

To test the differences between the experimental conditions, data points across time were reduced to three: (1) the mean of the four control condition values, (2) the last of the two transition values, and (3) the last of 10 hypoxia values. Comparisons among these three means were made following Fisher's protected $F$ (or $t$ ) procedure, which consisted of an overall test of differences based on repeated-measures ANOVA followed, if significant, by paired Student's $t$ tests. The method of random regression modeling (Littell et al., 1996) was used to test for differential linear and quadratic time trends in the hypoxia phase between the unexposed and prenatally exposed groups. This method is essentially a variation of repeated-measures ANOVA and was necessitated by the occurrence of seven missing values (attributable to the absence of bursting) in the prenatal nicotine inspiratory GABAergic IPSC frequency group. The missing values are assumed to be missing at random. With this method, differences of interest between the trend lines would be manifested by either a group main effect (overall mean difference), a linear $\times$ group interaction (difference in linear trend), or a quadratic $\times$ group interaction (difference in quadratic curvature trend). Random regression model tests were calculated in SAS software version 8.2 using Proc Mixed with type I (sequential) tests. $p<0.05$ indicated significant differences. Unless otherwise indicated, all $p$ values are the result of a statistical comparison against control values using Fisher's protected $F$ procedure. Software used for statistics was GraphPad Prism 4.01 (Graphpad Software, San Diego, CA), SAS 8.2 (Cary, NC), Microcal Origin 6.0 (OriginLabs, Northhampton, MA), and Microsoft Excel (Microsoft, Redmond, WA).

\section{Results}

\section{$\mathrm{pH}$ profile of the thick medullary slice}

In seven slices, the mean $\mathrm{pH}$ at the upper surface of the tissue was $7.35 \pm 0.05 . \mathrm{pH}$ progressively decreased as the electrode was advanced through the tissue, reaching a relative minimum of $7.19 \pm 0.09$ at a depth of $384 \mu \mathrm{m}$. After this point, $\mathrm{pH}$ progressively increased to $7.34 \pm 0.05$ at the lower surface of the tissue (Fig. 1a, top trace).

When the same slices were perfused with hypoxic aCSF for 10 $\min , \mathrm{pH}$ at the upper surface of the tissue was $7.35 \pm 0.08$. $\mathrm{pH}$ progressively decreased as the electrode was advanced through the tissue, reaching a relative minimum of $7.14 \pm 0.03$ at a depth of $363 \mu \mathrm{m}$. After this point, $\mathrm{pH}$ progressively increased to $7.32 \pm$ 0.07 at the lower surface of the tissue (Fig. 1a, bottom trace). Mean $\mathrm{pH}$ values at both the surface and at the core of the slice under hypoxic conditions were not significantly different from the corresponding $\mathrm{pH}$ values under control conditions $(p>0.05$ using paired Student's $t$ tests).

\section{$\mathrm{pO}_{2}$ profile of the thick medullary slice}

$\mathrm{pO}_{2}$ at the upper surface of the tissue was $583 \pm 16$ Torr. $\mathrm{pO}_{2}$ progressively decreased as the electrode was advanced through the tissue, reaching a relative minimum of $157 \pm 37$ Torr at a depth of $345 \mu \mathrm{m}$. After this point, $\mathrm{pO}_{2}$ progressively increased to $594 \pm 31$ Torr at the lower surface of the tissue (Fig. $1 a$, top trace).

After perfusion for $10 \mathrm{~min}$ with hypoxic aCSF, $\mathrm{pO}_{2}$ at the upper surface of the tissue was $142 \pm 9.2$ Torr. $\mathrm{pO}_{2}$ progressively decreased as the electrode was advanced through the tissue, reaching a relative minimum of $0 \pm 0$ Torr at a depth of $368 \mu \mathrm{m}$. After this point, $\mathrm{pO}_{2}$ progressively increased to $155 \pm 12$ Torr at the lower surface of the tissue (Fig. $1 b$, top trace).

\section{Time course of $\mathrm{pO}_{2}$ changes during hypoxia insult}

At a depth of $300 \mu \mathrm{m}$ beneath the upper surface of the tissue, average $\mathrm{pO}_{2}$ during the $4 \mathrm{~min}$ control period was $163.3 \pm 12.9$ Torr. Immediately after the perfusate was changed to hypoxic media, $\mathrm{pO}_{2}$ rapidly declined, reaching the hypoxic threshold of 23 Torr in $\sim 2$ min. $\mathrm{pO}_{2}$ levels reached a steady state of $0.8 \pm 0.7$ Torr within the next $2 \mathrm{~min}$ and remained stable for the remaining minutes the slice was perfused with hypoxic aCSF (Fig. 1c). After $10 \mathrm{~min}$ of perfusion with hypoxic aCSF, the perfusate was switched back to the original, hyperoxic perfusate. $\mathrm{pO}_{2}$ then gradually increased, exceeding the hypoxic threshold $2 \mathrm{~min}$ and $15 \mathrm{sec}$ after the hyperoxic media was started. After $10 \mathrm{~min}$ of recovery, $\mathrm{pO}_{2}$ had returned to control levels (156.7 \pm 10.9 Torr).

\section{Respiratory activity in the thick medullary slice}

As seen in other similar medullary respiratory slice preparations, the frequency of respiratory-related hypoglossal discharge is significantly lower than in vivo preparations likely because of the reduced temperature of the preparation and the absence of sensory input to the medulla (Rekling and Feldman, 1998). Like in vivo preparations, when subjected to hypoxic challenge, respiratory-related activity in thick medullary slices also responds in a biphasic manner: the frequency of respiratory-related activity initially increases and subsequently decreases as it shifts to a gasping pattern (Telgkamp and Ramirez, 1999; Lieske et al., 2000).

Hypoxia caused a small but not statistically different increase in respiratory frequency (Control, $2.5 \pm 0.2$ bursts/min; Transition, $2.7 \pm 0.3$ bursts/min) followed by a significant decrease in respiratory burst frequency (Hypoxia, $1.3 \pm 0.2$ bursts/min; $p<$ $0.001 ; n=26$ preparations). In addition, hypoxia caused a significant decrease in the duration of inspiratory bursts (Control, $2.7 \pm 0.3 \mathrm{sec}$; Hypoxia, $1.4 \pm 0.2 \mathrm{sec} ; p<0.0001)$. Prenatal nicotine exposure did not significantly alter the frequency $(2.5 \pm$ 0.5 bursts $/ \mathrm{min})$ or duration $(3.3 \pm 0.3 \mathrm{sec})$ of respiratory-related hypoglossal discharge compared with unexposed animals.

\section{Effect of hypoxia on spontaneous and inspiratory inhibitory synaptic inputs to CVNs \\ Glycine}

To determine the effects of hypoxia on the frequency of glycinergic IPSCs in CVNs, glycinergic neurotransmission was isolated by 
a

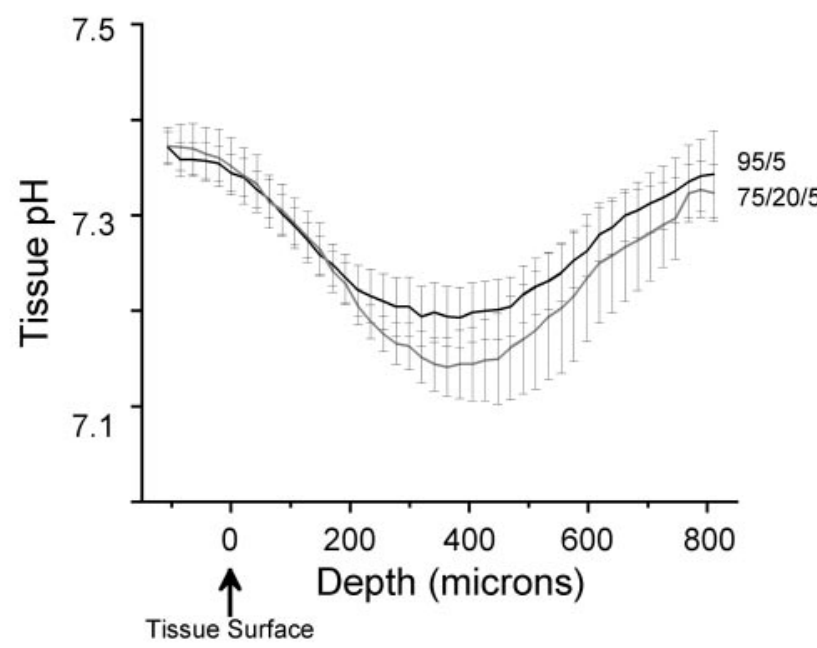

b
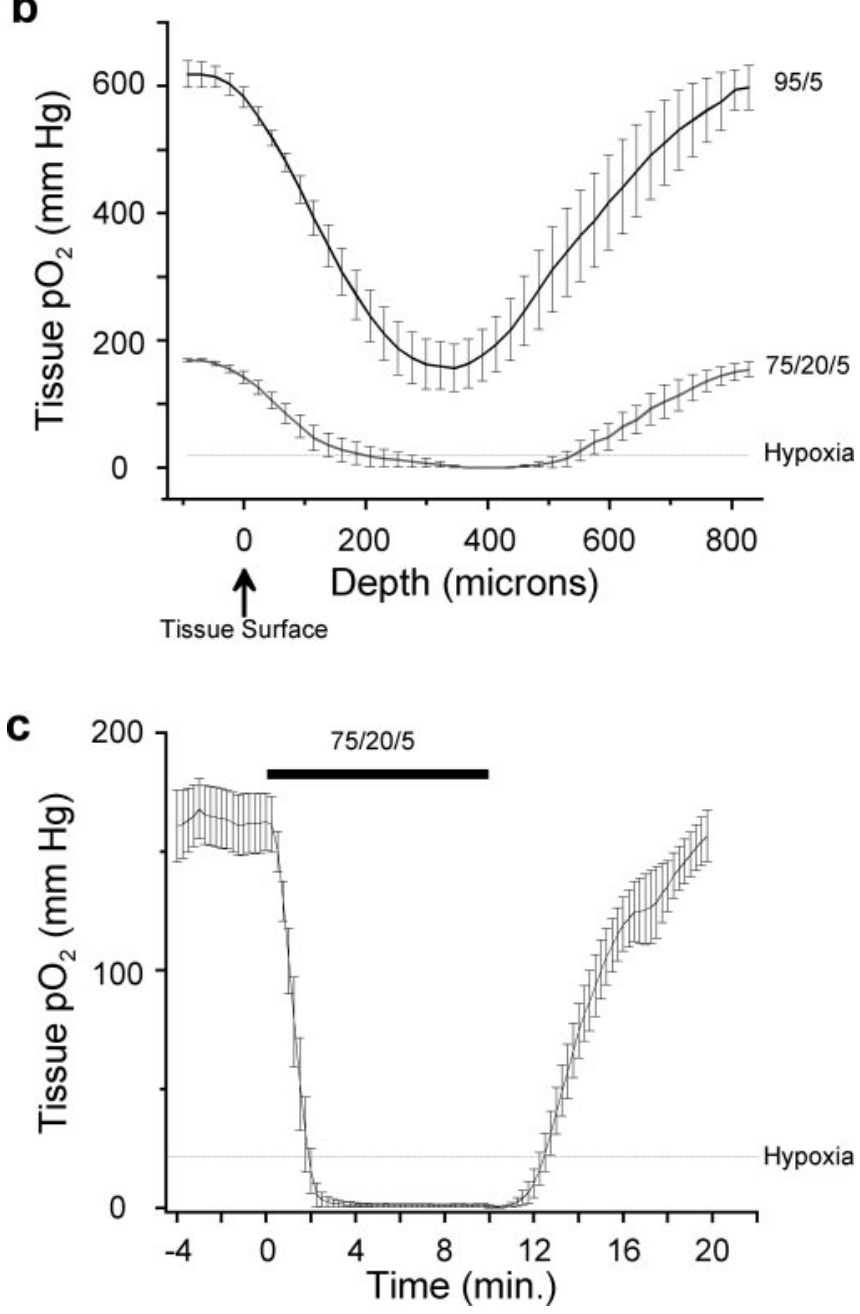

Figure 1. $\mathrm{pH}$ and $\mathrm{p}_{2}$ profiles of the thick medullary slice when the perfusate was saturated with a $95 \% \mathrm{O}_{2}-5 \% \mathrm{CO}_{2}$ gas mixture and when the perfusate was saturated with $75 \% \mathrm{~N}_{2}-20 \%$ $\mathrm{O}_{2}-5 \% \mathrm{CO}_{2}$ to evoke hypoxia. $a$, Top trace, $\mathrm{pH}$ progressively declined from the upper surface of the tissue to a relative minimum in the slice core. After this point, $\mathrm{pH}$ progressively increased as the electrode was advanced to the lower surface of the tissue $(n=7)$. Bottom trace, $\mathrm{pH}$ profile of the thick medullary slice under hypoxic conditions $(n=7) . b$, Top trace, $\mathrm{p}_{2}$ progressively declined from the upper surface of the tissue to a relative minimum in the slice core. After this point, $\mathrm{pO}_{2}$ progressively increased as the electrode was advanced to the lower surface of the tissue $(n=7)$. Bottom trace, $\mathrm{pO}_{2}$ profile of the thick medullary slice under hypoxic conditions the application of focal GABAergic and glutamatergic antagonists. During inspiratory bursts, glycinergic IPSC frequency significantly increased from $9.2 \pm 2.3$ to $14.6 \pm 2.9 \mathrm{~Hz}(p<0.01$; paired Student's $t$ test; $n=13$ cells) (Fig. $2 a$ ). Spontaneous glycinergic IPSC frequency responded to hypoxia in a biphasic manner, significantly increasing during the transition from control to hypoxia and then decreasing in the late stages of hypoxia (Control, $9.2 \pm 2.3 \mathrm{~Hz}$; Trans, $12.4 \pm 2.2 \mathrm{~Hz}, p<0.01$; Hypoxia, $6.5 \pm 0.9 \mathrm{~Hz}, n=13, p>0.05$ ) (Fig. $2 a, b$ ). Inspiratory glycinergic IPSC frequency also significantly increased and then significantly decreased during hypoxia (Control, $14.6 \pm 2.9 \mathrm{~Hz}$; Trans, $16.5 \pm 2.7, p<0.05$; Hypoxia, $9.3 \pm 1.3 \mathrm{~Hz}, n=13, p<0.05)$ (Fig. 2c).

Although spontaneous glycinergic IPSC frequency during hypoxia was not significantly different from the IPSC frequency under control conditions, spontaneous glycinergic IPSC frequency during hypoxia was significantly diminished from the glycinergic IPSC frequency during the transition phase $(p<$ $0.05)$. Despite this decrease, inspiratory glycinergic IPSC frequency remained statistically greater than spontaneous glycinergic IPSC frequency during hypoxia. All IPSCs under these recording conditions were reversibly inhibited by the focal application of the glycinergic antagonist strychnine.

\section{$G A B A$}

To determine the effects of hypoxia on the frequency of both spontaneous and inspiratory GABAergic IPSCs in CVNs, GABAergic neurotransmission was isolated by focal application of glycinergic and glutamatergic antagonists. During inspiratory bursts, the mean frequency of GABAergic IPSCs in CVNs significantly increased from $8.5 \pm 1.1 \mathrm{~Hz}$ to a frequency of $13.0 \pm 1.3$ $\mathrm{Hz}$ during inspiratory activity ( $p<0.01$; paired Student's $t$ test; $n=13$ cells) (Fig. $3 a$ ).

As shown in Figure 3, $a$ and $b$, spontaneous GABAergic IPSC frequency also changed in a biphasic manner during hypoxia, significantly increasing and then significantly decreasing (Control, $8.5 \pm 1.1 \mathrm{~Hz}$; Trans, $12.1 \pm 1.4 \mathrm{~Hz}, p<0.01$; Hypoxia, $5.1 \pm$ $1.0 \mathrm{~Hz}, p<0.05, n=13$ cells). Inspiratory GABAergic IPSC significantly decreased during hypoxia, but the transient increase during the transition phase was not statistically significant (Control, $13.11 \pm 1.4 \mathrm{~Hz}$; Transition, $15.3 \pm 1.5 \mathrm{~Hz}$; Hypoxia, $9.8 \pm$ $1.4 \mathrm{~Hz} ; p<0.05 ; n=13$ cells) (Fig. $3 a, c$ ). In the final minute of hypoxia, inspiratory GABAergic IPSC frequency was no longer significantly different from spontaneous GABAergic frequency. All IPSCs under these recording conditions were reversibly blocked by the application of the $\mathrm{GABA}_{\mathrm{A}}$ antagonist gabazine.

\section{Effect of prenatal nicotine on hypoxia-induced changes in spontaneous and inspiratory inputs to CVNs \\ Glycine}

Prenatal nicotine exposure did not significantly alter spontaneous or inspiratory glycinergic IPSC frequency under control conditions ( $n=13$ cells; $p>0.05$; unpaired Student's $t$ test). During hypoxia, changes in both spontaneous and inspiratory glycinergic IPSC frequency were similar to the biphasic responses seen in unexposed animals. Spontaneous glycinergic IPSC frequency ini-

\footnotetext{
$\leftarrow$

$(n=6)$. c, Time course of $\mathrm{pO}_{2}$ changes in the thick medullary slice at a depth of $300 \mu \mathrm{m}$. Perfusion with hypoxic media caused a rapid drop in $\mathrm{pO}_{2}$, which dropped below the hypoxic threshold in $\sim 2$ min and reached a steady state shortly thereafter $(n=7)$. The interval between the start of hypoxic perfusate (at time 0 ) and $\mathrm{p}_{2}$ dropping below the hypoxic threshold was defined as transition (Trans). Error bars represent SEM.
} 
a
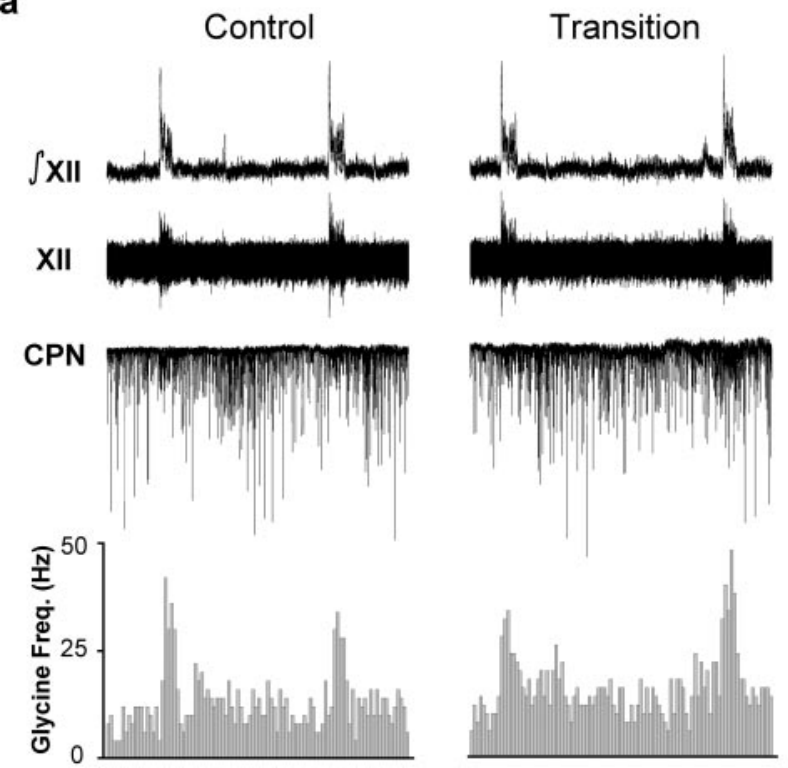

b

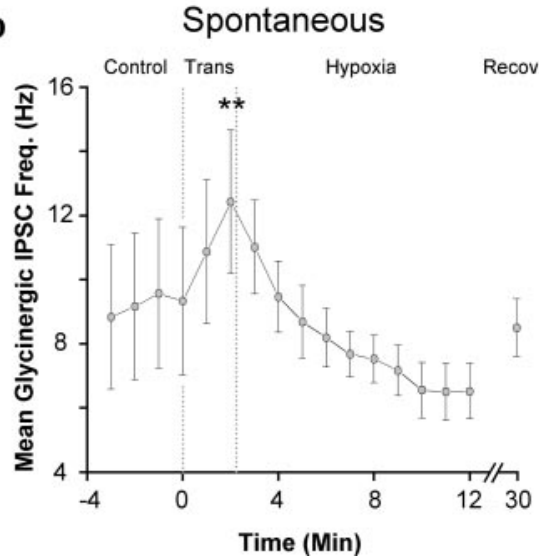

c

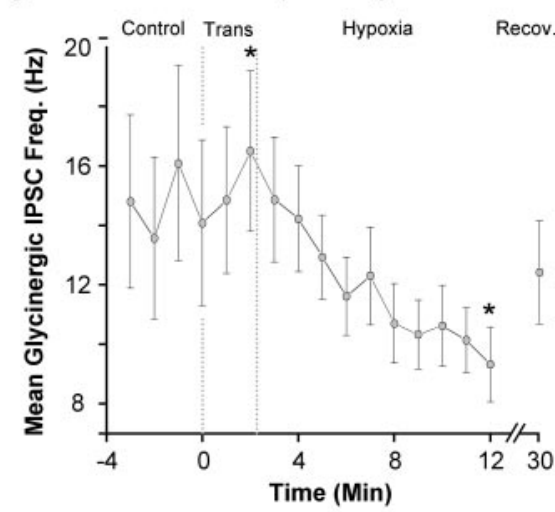

Figure 2. Hypoxia causes a biphasic change in glycinergic IPSC frequency. $a$, Inspiratory-related bursting activity was recorded from the hypoglossal rootlet (XII) and electronically integrated ( $\left.\int X I I\right)$. Fluorescently identified CVNs were patch clamped in the whole-cell configuration, and glycinergic neurotransmission was isolated by focal application of NMDA, non-NMDA, and GABA receptor antagonists. The frequency of glycinergic IPSC in CVNs was significantly increased during inspiratory bursts $(n=13 ; p<$ 0.01 using a paired Student's $t$ test). Hypoxia induced a significant increase in the frequency of spontaneous IPSCs in CVNs during the transition to hypoxia. Spontaneous glycinergic IPSC frequency was also significantly reduced during hypoxia relative to glycinergic IPSC frequency during the transition to hypoxia $(p<0.05 ; n=13)$. Inspiratory glycinergic IPSC frequency also transiently increased during transition ( $p<0.05$ ) and subsequently significantly decreased during hypoxia ( $p<0.05 ; n=13$ ). $b$, Average spontaneous glycinergic IPSC frequency \pm SE. c, Average inspiratory glycinergic IPSC frequency \pm SE. ${ }^{*} p<0.05$; ${ }^{* *} p<0.01$. All statistical comparisons were performed using Fisher's protected $F$ procedure unless otherwise indicated.

tially increased and subsequently decreased during hypoxic insult (Control, $11.8 \pm 2.5 \mathrm{~Hz}$; Transition, $16.0 \pm 2.4 \mathrm{~Hz}$; Hypoxia, $6.0 \pm 1.0 \mathrm{~Hz})$. Although these changes were not statistically significant $(p>0.05 ; n=7)$, spontaneous glycinergic IPSC frequency during hypoxia was significantly decreased compared with spontaneous glycinergic IPSC frequency during the transition to hypoxia ( $p<0.01 ; n=13$ unexposed; $n=7$ prenatal nicotine). Inspiratory glycinergic IPSC frequency also transiently increased and subsequently decreased during hypoxia (Control, $15.4 \pm 1.9 \mathrm{~Hz}$; Transition, $17.2 \pm 2.3 \mathrm{~Hz}$; Hypoxia, $11.8 \pm 0.5$ $\mathrm{Hz})$. However, none of these changes were statistically significant $(p>0.05 ; n=7)$.

To compare the changes in the frequency of glycinergic IPSCs in CVNs that occur in response to hypoxia in unexposed animals versus CVNs from animals exposed to nicotine prenatally, ran- dom regression modeling analysis was performed. This analysis revealed that for both spontaneous and inspiratory glycinergic IPSC frequency, group differences were not significant. However, group $\times$ quadratic time trend interactions were statistically significant, with fitted lines showing that the frequency of spontaneous and inspiratory glycinergic IPSCs, which were initially nonsignificantly elevated in prenatal nicotine CVNs, converged with the spontaneous and inspiratory glycinergic frequencies of unexposed CVNs during hypoxia (Spontaneous, $p<0.01$; Inspiratory, $p<0.05 ; n=13$ unexposed; $n=7$ prenatal nicotine). All IPSCs under these recording conditions were reversibly blocked by the focal application of the glycinergic antagonist strychnine.

GABA

GABAergic IPSC frequency was significantly increased during inspiration in animals exposed to nicotine prenatally (Basal, $10.0 \pm 1.7 \mathrm{~Hz}$; Inspiration, $19.8 \pm 3.3 \mathrm{~Hz}$; $n=10$ cells; $p<0.01$; paired Student's $t$ test) (Fig. $4 a$ ). In agreement with previous work (Neff et al., 2003), the inspiratoryrelated increase in GABAergic IPSC frequency during inspiration was significantly enhanced in CVNs from animals exposed to nicotine prenatally relative to unexposed animals (Unexposed, $13.0 \pm$ $1.3 \mathrm{~Hz}, n=13$ cells; Prenatal Nicotine, $19.8 \pm 3.3 \mathrm{~Hz}, n=10$ cells; $p<0.01$; unpaired Student's $t$ test).

In animals exposed to nicotine prenatally, the response of spontaneous GABAergic frequency was not biphasic. Rather, spontaneous GABAergic IPSC frequency precipitously decreased (Control, $10.0 \pm 1.7 \mathrm{~Hz}$; Hypoxia, $4.3 \pm 1.3 \mathrm{~Hz} ; n=$ 10 cells; $p<0.01$ ) (Fig. 4a,b). Likewise, inspiratory GABAergic IPSC frequency was also significantly decreased during hypoxia in animals prenatally exposed to nicotine (Control, $19.8 \pm 2.9 \mathrm{~Hz}$; Hypoxia, $9.2 \pm 2.2 \mathrm{~Hz} ; n=10$ cells; $p<0.01$ )

(Fig. 4c). All IPSCs under these recording conditions were reversibly blocked by the application of the $\mathrm{GABA}_{\mathrm{A}}$ antagonist gabazine.

To illustrate the major differences between the responses of unexposed and prenatal nicotine-exposed animals to hypoxia, the responses of these two groups are plotted together in Figure 4, $d$ and $e$. In CVNs from animals exposed to nicotine prenatally, spontaneous GABAergic IPSC frequency was not significantly different from unexposed animals before hypoxia (Unexposed, $8.5 \pm 1.1 \mathrm{~Hz}$; Prenatal Nicotine, $10.0 \pm 1.7 \mathrm{~Hz}$ ) (Fig. 4d). However, random regression model testing comparing the response of spontaneous GABAergic IPSC frequency to hypoxia in unexposed CVNs versus animals exposed to nicotine prenatally revealed that spontaneous GABAergic IPSC frequency in animals exposed to nicotine prenatally was significantly different from 
unexposed spontaneous GABAergic IPSC frequency on both a group main effect $(p<0.05)$ as well as a group $\times$ quadratic time trend interaction $(p<0.01 ; n=13$ unexposed cells; $n=10 \mathrm{PN}$ cells) (Fig. $4 d$ ). In CVNs exposed to nicotine prenatally, but not in unexposed CVNs, spontaneous GABAergic IPSC frequency fell precipitously in response to hypoxia. In contrast, although inspiratory GABAergic IPSC frequency was greater in animals exposed to nicotine prenatally compared with unexposed animals during control conditions, random regression model testing comparing the hypoxia response of inspiratory GABAergic IPSC frequency in unexposed CVNs versus inspiratory GABAergic IPSC frequency in CVNs from animals exposed to nicotine prenatally revealed no statistically significant differences in the inspiratory GABAergic IPSC responses to hypoxia between unexposed CVNs and CVNs exposed to nicotine prenatally (Fig. 4e).

\section{Discussion}

There are three major findings from this study: (1) The frequencies of both GABAergic and glycinergic IPSCs in CVNs change in a biphasic manner, transiently increasing and subsequently decreasing during hypoxia. (2) The response of spontaneous glycinergic IPSCs to hypoxia in CVNs exposed to nicotine prenatally remains biphasic, whereas inspiratory glycinergic IPSC frequency was not significantly altered during hypoxia. (3) Prenatal nicotine exposure abolishes the transient increase and significantly enhances the decrease in spontaneous GABAergic IPSCs in CVNs evoked during hypoxia.

Heart rate is primarily determined by the activity of brainstem CVNs in the NA (McAllen and Spyer, 1976; Machado and Brody, 1988; Loewy and Spyer, 1990; Mendelowitz, 1999). CVNs are intrinsically silent, and thus rely on synaptic inputs to determine their activity. Hyperpolarization of CVNs before depolarization relieves the voltage-dependent inactivation of 4-aminopyridine-sensitive potassium channels, which results in a significant delay before the initiation of action potentials in CVNs during subsequent depolarizations (Mendelowitz, 1996). Thus, hyperpolarizing synaptic inputs can inhibit CVNs both directly and by removing inactivation of this $\mathrm{K}^{+}$current, allowing inhibitory synaptic inputs to play a substantial role in dictating CVN activity and heart rate.

This study demonstrates that hypoxia elicits a biphasic change in the frequency of both GABAergic IPSCs and glycinergic IPSCs in CVNs: an initial increase followed by a decrease. The initial inhibition of CVNs would result in a tachycardia caused by a withdrawal of parasympathetic activity. The subsequent decrease in GABAergic IPSC frequency would elicit a bradycardia attributable to increased parasympathetic outflow to the heart.

Although the pattern of changes in the frequency of spontaneous glycinergic IPSCs in CVNs during hypoxia mimicked the
Control Transition
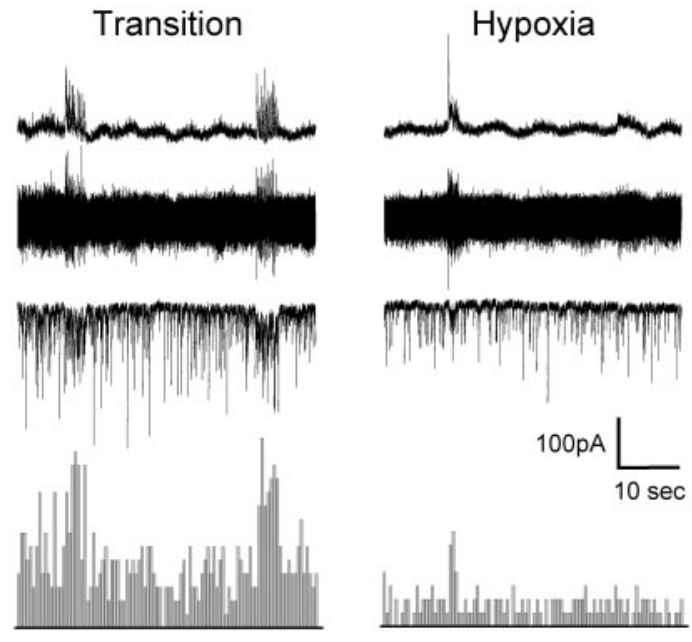

b

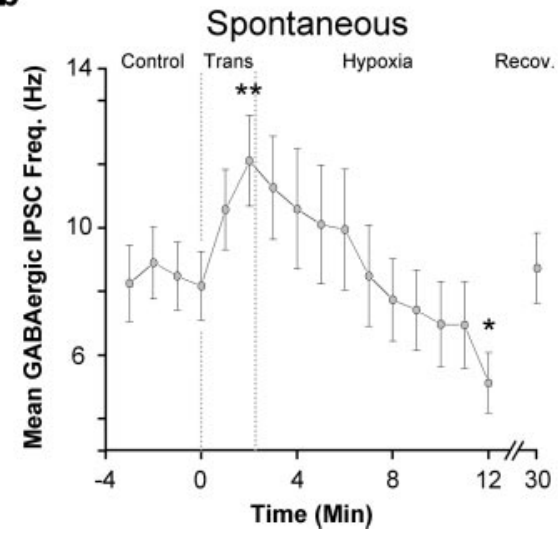

C

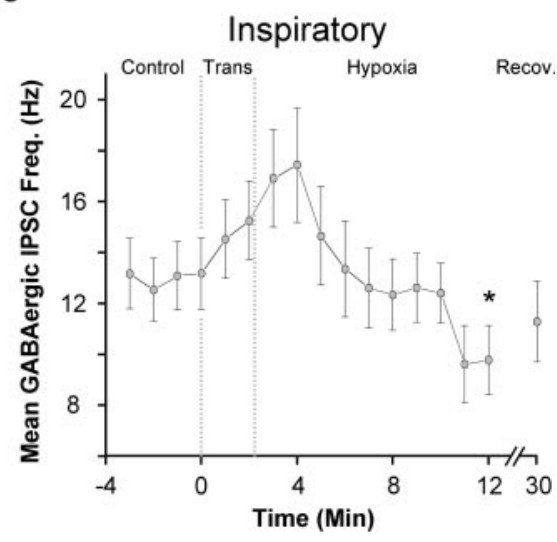

Figure 3. Hypoxia induces a biphasic change in GABAergic IPSC frequency in CVNs. GABAergic synaptic activity was isolated by focal application of the NMDA, non-NMDA, and glycinergic antagonists AP-5 (50 $\mu \mathrm{mol} / \mathrm{l})$, CNQX (50 $\mu \mathrm{mol} / \mathrm{l})$, and strychnine (1

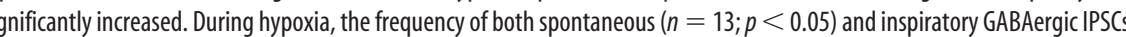
inspiratory GABAergic IPSC frequency \pm SE. ${ }^{*} p<0.05 ;{ }^{* *} p<0.01$. All statistical comparisons were performed using Fisher's protected $F$ procedure unless otherwise indicated.

hypoxia-induced changes in spontaneous GABAergic IPSC frequency, the decrease in the frequency of spontaneous glycinergic IPSCs in CVNs during hypoxia was not statistically different from control values. However, the frequency of spontaneous glycinergic IPSCs during hypoxia was significantly decreased compared with the elevated glycinergic IPSC frequency during the transition to hypoxia, suggesting that although spontaneous glycinergic activity may play a role in generating the heart rate changes during hypoxia, it seems likely that spontaneous GABAergic IPSCs in CVNs have a greater role in producing hypoxia-induced bradycardia.

Hypoxia caused a significant decrease in the frequency of inspiratory-related glycinergic IPSCs. The frequency of inspiratoryrelated GABAergic IPSCs in CVNs was also significantly decreased during hypoxia. These decreases in the GABAergic and glycinergic inspiratory-related inhibition of CVNs would result in a decreased inspiratory-related tachycardia. This may be a cellular basis for the reduction in respiratory sinus arrhythmia that is observed during hypoxia in some in vivo studies (Yasuma et al., 2001).

Both GABAergic and glycinergic neurotransmission to CVNs 


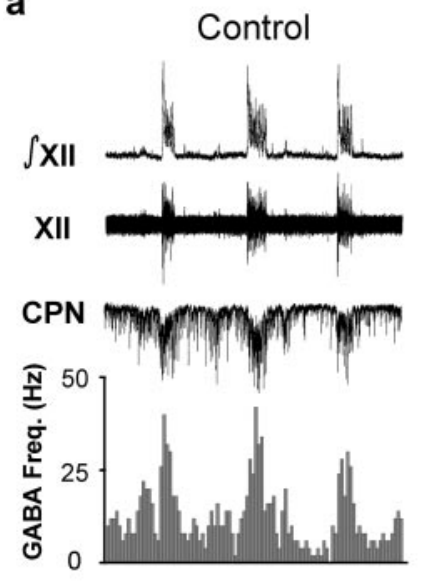

b

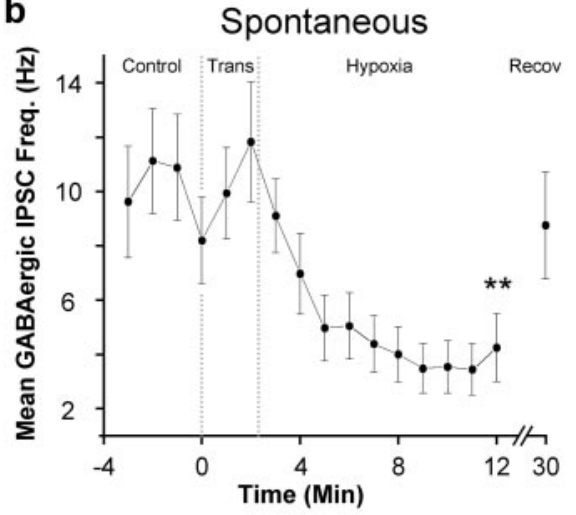

d

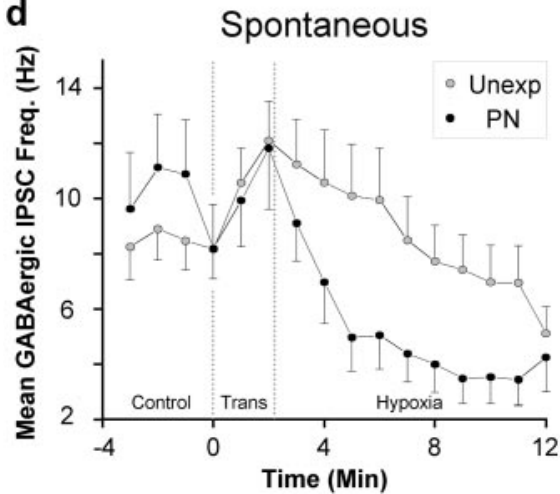

Transition
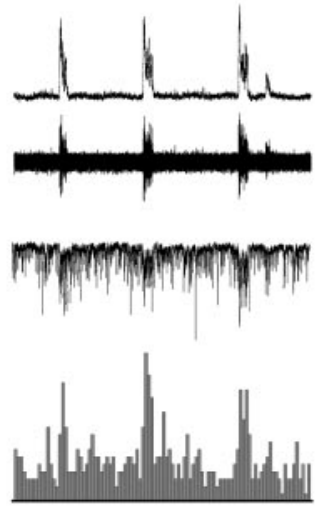

C

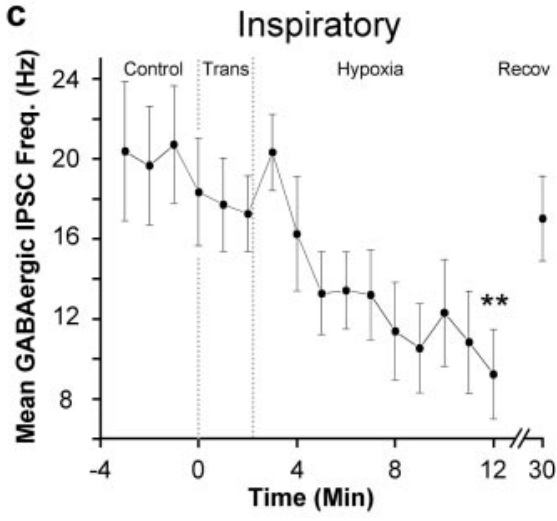

e

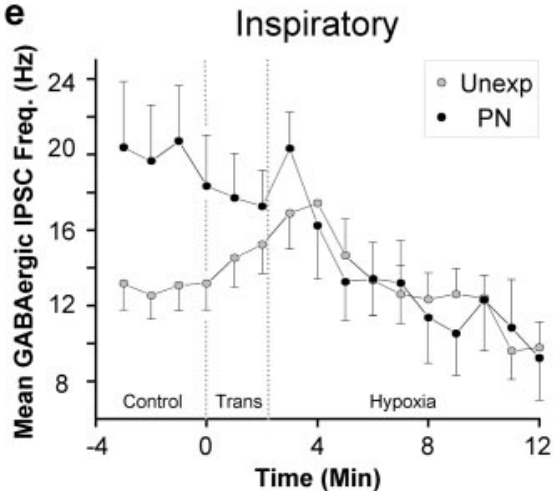

Figure 4. Hypoxia induces a rapid decrease in the frequency of both spontaneous and inspiratory GABAergic IPSCs in CVNs from animals exposed to nicotine prenatally. $a$, In animals prenatally exposed to nicotine, the inspiratory-related increase in GABAergic IPSC frequency was significantly increased compared with control animals ( $n=13$ Unexposed; $n=10$ Prenatal Nicotine; $p<$ 0.05 using an unpaired Student's $t$ test). $b, c$, Hypoxia induced a rapid decrease in the frequency of both spontaneous and inspiratory GABAergic IPSCs in CVNs. Values are mean GABAergic IPSC frequency \pm SE. Double asterisks denote $p<0.01$ versus mean control frequency. $d$, During hypoxia, the frequency of spontaneous GABAergic IPSCs in CVNs in animals exposed to nicotine prenatally was significantly lower than the frequency of spontaneous GABAergic IPSCs in unexposed animals (random regression model testing group main effect, $p<0.05$; group $\times$ quadratic time trend interaction, $p<0.005 ; n=13$ unexposed CVNs; $n=$ 10 PN CVNs).e, Under control conditions, the frequency of inspiratory GABAergic IPSCs in CVNs was significantly greater in animals prenatally exposed to nicotine compared with unexposed animals (unpaired Student's $t$ test; $p<0.01$ ). Hypoxia abolished the elevation of inspiratory GABAergic IPSC frequency in animals prenatally exposed to nicotine (random regression model testing group main effect, $p>0.05$; group $\times$ quadratic time trend interaction, $p>0.05$ ).

is modulated by endogenous activation of presynaptic $\alpha 4 \beta 2$ nicotinic receptors (Wang et al., 2003). Nicotinic receptor function is known to be suppressed during hypoxia (Lee et al., 1995). Diminished nicotinic receptor function during hypoxia would result in a decreased level of GABAergic and glycinergic neurotransmission to CVNs during hypoxia and could account for the hypoxia-induced decrease in the frequency of GABAergic and glycinergic IPSCs in CVNs.

These cellular changes are highly predictive for what is observed in vivo. Hypoxia causes a transient tachycardia followed by a parasympathetically mediated bradycardia in vivo (Guntheroth and Kawabori, 1975; Taylor and Butler, 1982; Loewy and Spyer, 1990; Schuen et al., 1997; Slotkin et al., 1997; Deshpande et al., 1999; Gozal et al., 2002). It is important to note that the time course of the changes in spontaneous glycinergic and GABAergic IPSC frequencies in CVNs is strikingly similar to the time course of hypoxiainduced heart rate changes in neonatal rats (Slotkin et al., 1997). The strong similarity of these responses suggests that alterations in spontaneous glycinergic and GABAergic inputs to CVNs play a critical role in dictating parasympathetic tone during hypoxia.

In animals exposed to nicotine prenatally, the hypoxia response of spontaneous glycinergic IPSCs in CVNs remained biphasic. Inspiratory glycinergic IPSC frequency did not significantly change during hypoxia. This suggests that with prenatal nicotine exposure, the pathways that mediate the increase in glycinergic neurotransmission in CVNs during inspiration are resistant to hypoxia. However, compared with unexposed animals, prenatal nicotine exposure did not significantly alter spontaneous or inspiratory glycinergic IPSC frequency responses to hypoxia by overall mean or by trend. This is not surprising given that in previous studies, glycinergic IPSCs in CVNs were not affected by prenatal nicotine exposure (Neff et al., 2003).

In contrast, prenatal nicotine exposure abolished the initial hypoxia-induced increase in spontaneous GABAergic IPSC frequency that occurred in unexposed animals and caused a greater and more rapid reduction in spontaneous GABAergic frequency during hypoxia. In addition, the enhanced increase in GABAergic IPSC frequency during inspiration caused by prenatal nicotine exposure was rapidly abolished during hypoxia. An exaggerated disinhibition of CVNs would induce a more rapid increase in parasympathetic outflow to the heart and a bradycardia in vivo.

In animals exposed to nicotine prenatally, the time course of the changes in spontaneous GABAergic IPSC frequency observed in this study also very closely mimics the heart rate changes observed in in vivo experiments using animals that have been exposed to nicotine prenatally (Slotkin et al., 1997). Compared with unexposed animals, animals prenatally exposed to nicotine re- 
spond to hypoxia with a greater decrease in heart rate. This impaired heart rate control reduces hypoxia tolerance in neonatal rats and has been hypothesized to be the mechanism which accounts for the relationship between maternal smoking and SIDS (Meny et al., 1994; Slotkin et al., 1997; Poets et al., 1999). The enhanced hypoxia-induced withdrawal of GABAergic neurotransmission in animals exposed to nicotine prenatally provides a likely neurochemical mechanism for the substantial and potentially lethal exaggeration of the hypoxia-induced bradycardia observed in rats prenatally exposed to nicotine and a cellular mechanism by which prenatal nicotine could increase SIDS incidence.

In summary, CVNs are transiently inhibited and subsequently disinhibited during hypoxia by an increase, followed by a decrease, in the frequency of spontaneous GABAergic and glycinergic IPSCs. This alteration in inhibitory neurotransmission likely plays a critical role in mediating the hypoxia-induced protective slowing of the heart. Inspiratory-related GABAergic and glycinergic neurotransmission to CVNs is diminished during hypoxia. This hypoxia-induced decrease in the inspiratory-related inhibition of CVNs may account for the diminished RSA that can occur during hypoxia. Prenatal nicotine exposure changes the GABAergic response to hypoxia from a biphasic increase and subsequent decrease in GABAergic IPSC frequency to a more severe decrease in GABAergic IPSC frequency. These data establish a likely neurochemical mechanism for the heart rate response to hypoxia, provide a link between prenatal nicotine exposure and an exaggerated bradycardia during hypoxia, and supply a potential cellular foundation for the link between prenatal nicotine exposure and SIDS.

\section{References}

Anderson RN (2002) Deaths: leading causes for 2000. Natl Vital Stat Rep 50:1-85.

Bamford O, Carroll J (1999) Dynamic ventilatory responses in rats: normal development and effects of prenatal nicotine exposure. Respir Physiol 117:29-40.

Deshpande P, Khurana A, Hansen P, Wilkins D, Thach BT (1999) Failure of autoresuscitation in weanling mice: significance of cardiac glycogen and heart rate regulation. J Appl Physiol 87:203-210.

Doppenberg EM, Zauner A, Watson JC, Bullock R (1998) Determination of the ischemic threshold for brain oxygen tension. Acta Neurochir Suppl (Wien) 71:166-169.

Fewell JE, Smith FG, Ng VK (2001) Prenatal exposure to nicotine impairs protective responses of rat pups to hypoxia in an age dependent manner. Respir Physiol 127:61-73.

Gozal D, Gozal E, Reeves SR, Lipton AJ (2002) Gasping and autoresuscitation in the developing rat: effect of antecedent intermittent hypoxia. J Appl Physiol 92:1141-1144.

Guntheroth WG, Kawabori I (1975) Hypoxia apnea and gasping. J Clin Invest 56:1371-1377.

Hunt CE (2001) Sudden infant death syndrome and other causes of infant mortality: diagnosis, mechanisms, and risk for recurrence in siblings. Am J Respir Crit Care Med 164:346-357.

Lee K, Ito A, Koshimura K, Ohue T, Takagi Y, Miwa S (1995) Differential effects of hypoxia on ligand binding properties of nicotinic and muscarinic acetylcholine receptors on cultured bovine adrenal chromaffin cells. J Neurochem 64:874-882.

Lieske SP, Thoby-Brisson M, Telgkamp P, Ramirez JM (2000) Reconfiguration of the neural network controlling multiple breathing patterns: eupnea, sighs, and gasps. Nature Neurosci 3:600-607.
Littell RC, Milliken GA, Stroup WW, Wolfinger RD (1996) SAS system for mixed models. Cary, NC: SAS Institute.

Loewy AD, Spyer KM (1990) Central regulation of autonomic functions. New York: Oxford UP.

Machado BH, Brody MJ (1988) Role of the nucleus ambiguus in the regulation of heart rate and arterial pressure. Hypertension 11:602-607.

McAllen RM, Spyer KM (1976) The location of cardiac vagal preganglionic motoneurones in the medulla of the cat. J Physiol (Lond) 258:187-204.

Mendelowitz D (1996) Firing properties of identified parasympathetic cardiac neurons in nucleus ambiguus. Am J Physiol 271:H2609-H2614.

Mendelowitz D (1999) Advances in parasympathetic control of heart rate and cardiac function. News Physiol Sci 14:155-161.

Mendelowitz D, Kunze DL (1991) Identification and dissociation of cardiovascular neurons from the medulla for patch clamp analysis. Neurosci Lett 132:217-221.

Meny RG, Carroll JL, Carbone MT, Kelly DH (1994) Cardiorespiratory recordings from infants dying suddenly and unexpectedly at home. Pediatrics 93:44-49.

Nachmanoff DB, Panigrahy A, Filiano JJ, Mandell F, Sleeper LA, ValdesDapena M, Krous HF, White WF, Kinney HC (1998) Brainstem 3Hnicotine receptor binding in the sudden infant death syndrome. J Neuropathol Exp Neurol 57:1018-1025.

Neff RA, Wang J, Baxi S, Evans C, Mendelowitz D (2003) Respiratory sinus arrhythmia: endogenous activation of nicotinic receptors mediates respiratory modulation of brainstem cardioinhibitory parasympathetic neurons. Circ Res 93:565-572.

Poets CF, Meny RG, Chobanian MR, Bonofiglo RE (1999) Gasping and other cardiorespiratory patterns during sudden infant deaths. Pediatr Res 45:350-354.

Rekling JC, Feldman JL (1998) PreBotzinger complex and pacemaker neurons: hypothesized site and kernel for respiratory rhythm generation. Annu Rev Physiol 60:385-405.

Schuen JN, Bamford OS, Carroll JL (1997) The cardiorespiratory response to anoxia: normal development and the effect of nicotine. Respir Physiol 109:231-239.

Slotkin TA (1998) Fetal nicotine or cocaine exposure: which one is worse? J Pharmacol Exp Ther 285:931-945.

Slotkin TA, Saleh JL, McCook EC, Seidler FJ (1997) Impaired cardiac function during postnatal hypoxia in rats exposed to nicotine prenatally: implications for perinatal morbidity and mortality, and for sudden infant death syndrome. Teratology 55:177-184.

Smith JC, Ellenberger HH, Ballanyi K, Richter DW, Feldman JL (1991) PreBotzinger complex: a brainstem region that may generate respiratory rhythm in mammals. Science 254:726-729.

St-John WM, Leiter JC (1999) Maternal nicotine depresses eupneic ventilation of neonatal rats. Neurosci Lett 267:206-208.

Taylor EW, Butler PJ (1982) Nervous control of heart rate: activity in the cardiac vagus of the dogfish. J Appl Physiol 53:1330-1335.

Telgkamp P, Ramirez JM (1999) Differential responses of respiratory nuclei to anoxia in rhythmic brain stem slices of mice. J Neurophysiol 82:2163-2170.

Wang J, Irnaten M, Mendelowitz D (2001) Characteristics of spontaneous and evoked GABAergic synaptic currents in cardiac vagal neurons in rats. Brain Res 889:78-83.

Wang J, Irnaten M, Venkatesan P, Evans C, Baxi S, Mendelowitz D (2002) Synaptic activation of hypoglossal respiratory motorneurons during inspiration in rats. Neurosci Lett 332:195-199.

Wang J, Wang X, Irnaten M, Venkatesan P, Evans C, Baxi S, Mendelowitz D (2003) Endogenous acetylcholine and nicotine activation enhances GABAergic and glycinergic inputs to cardiac vagal neurons. J Neurophysiol 89:2473-2481.

Yasuma F, Hirai M, Hayano J (2001) Differential effects of hypoxia and hypercapnia on respiratory sinus arrhythmia in conscious dogs. Jpn Circ J 65:738-742. 